\title{
MULTISYSTEM INFLAMMATORY SYNDROME ASSOCIATED SARS-COV-2. A NOVEL SPECTRUM OF PEDIATRIC ILNESS
}

\author{
Simona Cainap, Alexandra Mititelu, Oana Pantar, Diana Lazar \\ University of Medicine and Pharmacy"Iuliu Hatieganu" Cluj, \\ Clinical Emergency Hospital for Children, Second Department of Pediatrics
}

\begin{abstract}
Multisystem Inflammatory Syndrome associated with COVID-19 (MIS-C) has been described as a novel and often severe presentation of SARS-CoV-2 infection in children.

This syndrome is characterized by fever, abdominal pain, gastrointestinal and cutaneous symptoms, and hemodynamic alterations. MIS-C has similar features to those of Kawasaki disease (KD), toxic shock syndrome (TSS), macrophage-activation syndrome, bacterial sepsis, meningitis.

MIS-C is defined as a typically progressive disease. Patients who initially had mild symptoms can develop severe illness with multi-organ dysfunction a few days after symptom onset.

The majority of affected children were treated with intravenous immune globulin (IVIG), and several also received adjunctive high-dose steroids.

The pediatricians should be aware about this new and potentially life threatening condition in children during COVID-19 epidemics.
\end{abstract}

Key words: Covid-19, Multisystemic inflammatory syndrome, children

\section{Introduction}

Since the first cases that emerged in December 2019 in Wuhan, China, the coronavirus disease 2019 (COVID-19) has quickly spread all over the world. Until today, more than 130 million persons have been infected with the new coronavirus, with more than 2 million deaths from COVID-19 [1].

Although SARS-CoV-2 infects people of all ages, evidence from several studies suggests that children and adolescents may be less commonly infected with SARS-CoV-2 than adults. The available data collected so far show that individuals aged $\leq 17$ years represents only $1-8 \%$ of laboratory-confirmed cases of COVID-19, and only $2-4 \%$ of patients required hospital admission [2,3].

Around $90 \%$ of cases were asymptomatic or mild or moderate, with a good prognosis and a complete recovery within 1-2 weeks, and the risk of severe illness was considered very low $[4,5]$.

However, among severe cases, a previously unknown SARS-CoV-2-related clinical picture emerged, manifesting as a hyper-inflammatory syndrome, with multi-organ involvement, such as sepsis, toxic shock syndrome, myocarditis, and meningitis, macrophage activation syndrome, Kawasaki disease.

\section{Definition on MIS-C}

Since the first alert by the UK and initial descriptions around the world, different clinical classifications have been proposed by the World Health Organization, the UK Royal College of Pediatrics and Child Health, the French "COVID pediatric inflammation" consortium and the US Centre for Disease Control and Prevention [6].

* Correspondent author: Oana Pantar, University of Medicine and Pharmacy"Iuliu Hatieganu" Cluj, Clinical Emergency Hospital for Children, Second Department of Pediatrics, Cluj-Napoca, Romania, e-mail: oana_ong@ yahoo.com

Article received: 10.04 .2021 , accepted: 12.04 .2021 , published: 12.04 .2021

Cite: Cainap S, Mititelu A, Pantar O, Lazar D. Multisystem Inflammatory Syndrome associated SARS-CoV-2. A novel spectrum of pediatric ilness. The Journal of School and University Medicine 2021;VIII(1):36-40 
In late April 2020, a group of UK pediatricians, warned about a high number of cases in children with COVID-19 infection that was asymptomatic or minimally symptomatic who were diagnosed with a significant systemic inflammatory response with high fever. They also observed the involvement of more than one organ or body system with a severe evolution requiring hospitalization and a risk of death, although rare. The Royal College of Paediatrics and Child Health named this condition pediatric inflammatory multisystem syndrome associated temporally with severe acute respiratory syndrome coronavirus 2 (PIMS-TS) [7].

On May 14th, 2020 the United States Centers for Disease Control and Prevention (CDC) released a health advisory reporting a multisystem inflammatory syndrome in children (MIS-C) associated with COVID-19. This statement stemmed from a subset of pediatric patients manifesting with severe inflammation, multi-organ failure, and testing positive for SARS-CoV-2 [8].

The WHO has developed a similar preliminary case definition and a case report form for multisystem inflammatory disorder in children and adolescents [9].

\section{Pathogenesis}

The pathogenesis of COVID-19 is not completely understood but it is thought to be a post-infectious immune-mediated phenomenon.

The low positivity rate of direct testing, coupled with a high proportion of patients who were antibody positive, suggest that MIS-C is not viral-mediated but more likely to be a delayed dysregulated immune response to SARS-CoV-2 with massive pro-inflammatory production.

The innate immunity system is activated during SARS-CoV-2 infection, fact that leads to a "cytokine storm", characterized by persistent fever and markedly elevated cytokines mostly TNF- $\alpha$, IL-1RA, IL-6, IL-10, IL-17, IL-18, IFN - $\gamma$, M-CSF, G-CSF, IP-10, and MCP [10].

Current evidence reports SARS-CoV-2 may act as a superantigen. The mechanism is similar with the staphylococcal enterotoxin B (SEB) that binds to the $\mathrm{T}$ cell receptor (TCR) and the costimulatory molecule CD28, mediating TSS [11].

\section{Clinical presentations}

Cardinal signs are persistent fever, mucocutaneous changes (hands and feet oedema, conjunctivitis, swollen and cracked red lips, rash) gastrointestinal symptoms (eg, abdominal pain, vomiting, diarrhoea). The abdominal pain in MIS-C can be so severe that in several cases patients were presumed to have appendicitis. [12]

A significant percentage (35-100\%) associate cardiac dysfunction (myocarditis, arrhythmia, coronary aneurysm or dilation) with severe evolutionary potential. $[13,14]$

Critical signs are represented by tachycardia, haemodynamic instability, left ventricular dysfunction, and respiratory distress, that could be caused by cardiac dysfunction or could be primary. An important fact is that a subset of patients presents with hypotension and shock. This could be caused by acute myocardial involvement or by systemic hyperinflammation with or without vasodilation, frequently requiring admission in the intensive care units, providing respiratory and circulatory support. $[15,16]$

The presence of neurological (headache, irritability, and encephalopathy), hematological and renal manifestations is also noticeable. (Table 1)

Table 1 Clinical features of patients with MIS-C summarised from two surveillance studies and one meta-analysis( adapted by Rubens) [17]

\begin{tabular}{|l|c|}
\hline Organ system involvement & Range \\
\hline Gastrointestinal & $87-92 \%$ \\
\hline Cardiovascular & $67-87 \%$ \\
\hline Hematological & $74-76 \%$ \\
\hline Mucocutaneous & $71-74 \%$ \\
\hline Respiratory & $63-70 \%$ \\
\hline Neurological & $38-39 \%$ \\
\hline Admission to intensive care & $64-80 \%$ \\
\hline Mechanical ventilation & $13-30 \%$ \\
\hline Vasoactive support & $42-48 \%$ \\
\hline Death & $2-4 \%$ \\
\hline
\end{tabular}

Pacients begun to develop symptoms 2 to 4 weeks after infection with SARS-CoV-2, and in the majority of cases, COVID 19 disease had a mild course or had been asymptomatic. That is why many children can be unaware that they are infected. Moreover, current data shows that $52 \%$ of patients who 
developed the inflammatory syndrome did not have any underlying medical conditions.

\section{Laboratory and imaging tests}

When MIS-C is suspected, it is necessary to evaluate the level of inflammation, the function of the vital organs and to obtain additional diagnostic imaging. This is based on physical examination findings and laboratory results.

Patients with MIS C universally present significant elevation of inflammatory markers, such as C-reactive protein (CRP), erythrocyte sedimentation rate (ESR), procalcitonin, LDH and/or ferritin. [18] Inflammatory cytokine levels are elevated as well. Interleukin 6 (IL-6), tumor necrosis factor alpha (TNF $\alpha$ ), or IL10 are often elevated.[19].

Notably, hematologic abnormalities reported included neutrophilia, lymphopenia, low to normal platelet levels, elevated D-dimer, and low fibrinogen [20].

Other important findings are markedly elevated level of NT proBNP and high level of troponin.[21]

Hyponatremia, acute kidney injury, and hypoalbuminemia can occur as well.[18,20]

Echocardiography demonstrated depressed cardiac ventricular function in the majority of patients, dilated coronary arteries or frank coronary artery aneurysms, dysfunction, hypocontractility, or even segmental absence of contractility in the myocardial chambers.[21,22,23]

CXR: patchy symmetrical infiltrates/consolidation, atelectasis, pleural effusion.

CT chest findings: Nodular ground glass opacification.

Abdominal USS: colitis, ileitis, lymphadenopathy, ascites, hepatosplenomegaly;

\section{Diagnostic}

For the MIS-C diagnosis the following case definition criteria are recommended: [16]

- Presence of fever $>38$ grdC more than 24 hours

- Elevated inflammatory markers

- Multiorgan dysfunction(> 2systems: cardiac, dermatological, gastrointestinal, renal, respiratory, hematological, and/or neurological)
- Positive viral or serological testing for SAESCoV-2 or close contact with a person with Covid-19 within four weeks of symptoms onset

- No plausible alternative diagnosis

It is important to note that SARS-CoV-2 biological evidence is not required to define MIS-C.

MIS-C occurs 2-6 weeks after the acute episode of SARS-CoV-2 infection and is thought to overlap with peak antibody production. A large proportion of patients are already with a negative PCR test, presenting only antibodies against the virus.

\section{Treatment}

The management of patients with MIS-C requires a multidisciplinary team, including cardiology, rheumatology, intensive care, and infectious disease specialists, because of the multisystem nature. Treatment of patients with MIS-C often includes management of shock, the use of immunomodulatory therapies, and the use of thromboprophylaxis agents.

Patients with shock require immediate intervention beginning with fluid resuscitation, oxygen supplementation (including mechanical ventilation) and hemodynamic support as well( inotrope/or vasopressor drugs, ECMO).

Cardiac dysfunction requires vasoactive support in the majority of children, with mechanical ventilation instituted in approximately one third of children, and a small number requiring mechanical circulatory support.[12]

\section{Immunomodulatory therapy}

Because of the biochemical and clinical similarities with $\mathrm{KD}$, the principles of treatment for Kawasaki Disease were applied in the majority of patients, obtaining rapid clinical improvement and low levels of inflammatory markers in most patients.

The therapy recommended for all children is intravenous immunoglobulin. The standard IVIG dose is $2 \mathrm{~g} / \mathrm{kg}$, which is administered depending on the cardiac function, either in a single or divided dose. A second dose was often given if an inflammatory state persisted.[22,24] 
Second-line therapy is glucocorticoids:

- low-moderate dose glucocorticoids (1-2 $\mathrm{mg} / \mathrm{kg} /$ day) should be given with IVIG as adjunctive therapy for treatment of MIS-C in patients with shock and/or organ threatening disease $[22,24]$

- high dose, IV pulse glucocorticoids (10$30 \mathrm{mg} / \mathrm{kg} /$ day, $\max 1 \mathrm{~g}$ ) is considered if patient requires high dose or multiple inotropes and/or vasopressors or ongoing hyperpyrexia.

- Dexamethasone $10 \mathrm{mg} / \mathrm{m}^{2} \mathrm{qd}$ is considered in case of HLH or CNS involvement $[22,24]$

Biological therapy(tocilizumab, anakinra, infliximab) should be considered as a third-line option in children who do not respond to intravenous immunoglobulin and methylprednisolone. [25]

\section{Anticoagulation and antiplatelet treatment}

Anticoagulation and/or antiplatelet treatment is recommended considering the stasis from immobilization, ventricular dysfunction, coronary artery aneurysm, possible endothelial injury and the hypercoagulable state, conditions that increase the risk of thrombotic complications. [26]

Low dose aspirin (3-5 mg/kg/day; max 81 $\mathrm{mg} /$ day) should be used in patients with MIS-C and stopped when platelet count is back to normal and no coronary arteries abnormalities, at least four weeks after diagnosis. Aspirin should not be given in patients with active bleeding, significant bleeding risk, and/or platelet count $\leq 80,000 / \mu \mathrm{L}(\mathrm{M})$. [24]

Prophylaxis with LMWH is recommended based on D-dimer level (when D-Dimer is $>$ five times normal values and/or when other pro-thrombotic factors are known). [24]

Patients with MIS-C and documented thrombosis or a low ejection fraction (EF) should receive Enoxaparin $100 \mathrm{UI} / \mathrm{kg}$, two times a day. [24]

\section{Antiviral and antibiotic therapy}

When RT-PCR or antigen testing are positive for SARS-CoV-2 in a child with PIMS-TS, the antiviral therapy might be considered; the first-choice antiviral agent is Remdesivir [27].Until the microbiological culture results are available, presumed sepsis should be treated in all children with PIMS-TS. Intravenous, large spectrum antibiotics should be commenced in all patients (eg Ceftrioaxone, Ceftazidime, Vancomicine, Clindamicine) [27]

\section{Evolution. Prognosis}

Several unanswered questions remain regarding pathogenesis, long term complications or prognosis. Echocardiography is a key investigation during follow-up, due to the coronary artery aneurysms that have been appeared even after mild disease courses. After discharge, clinical follow-up is indicated at 1-2 weeks and 6 weeks.

\section{Conclusion}

Multisystem inflammatory syndrome is a new spectrum of pediatric disease that has emerged in association with SARS-CoV-2 infection.

Clinical symptoms are wide and non-specific but always feature high fever, multiorgan involvement, and strong biological inflammation. Although PIMS (or MIS-C ) occurs only in a small proportion of COVID-19 diseases, most of them require admission in intensive care units.

Management of acute MIS-C include immunomodulation, cardiac supportive therapy and antiplatelet/anticoagulation treatment.

Follow-up of MIS-C patients is a very important part of this disease, essential to better understand the evolution and prognosis.

\section{References}

1. Chengdi Wang, Zhoufeng Wang, Guangyu Wang, Johnson Yiu-Nam Lau, Kang Zhang, Weimin L. COVID-19 in early 2021: current status and looking forward. Signal Transduct Target Ther. 2021;6:114. Published online 2021 Mar 8. doi: 10.1038/s41392-021-00527-1

2. Lee PI, Hu YL, Chen PY, Huang YC, Hsueh PR. Are children less susceptible to COVID-19? J Microbiol Immunol Infect. 2020;53:371-2.

3. CDC COVID-19 Response Team. Coronavirus disease 2019 in children-United States, February 12-April 2, 2020. Morb. Mortal. Wkly. Rep. 2020, 69, 422-426.

4. Choi S-H, Kim HW, Kang J-M et al. Epidemiology and clinical features of coronavirus disease 2019 in children. Clin Exp Pediatr 2020; 63(4):125. https://doi.org/10.3345/cep.2020.00535

5. Dong Y, Mo X, Hu Y et al. Epidemiological characteristics of 2143 pediatric patients with 2019 coronavirus 
disease in China. J Emerg Med 2020;58(4):712-713. https://doi. org/10.1016/j. jemermed.2020.04.006

6. Riphagen S, Gomez X, Gonzalez-Martinez C, Wilkinson N, Theocharis P. Hyperinfammatory shock in children during COVID-19 pandemic. Lancet. 2020;395:1607-1608

7. Royal College of Paediatrics and Child Health. Guidance: paediatric multisystem inflammatory syndrome temporally associated with COVID-19.

https://www.rcpch.ac.uk/resources/guidancepaediatric-multisystem-inflammatory-syndrome-temporallyassociated-covid-19

8. Centers for Disease Control and Prevention. Emergency preparedness and response: health alert network. https://emergency.cdc.gov/ han/2020/han00432.asp

9. World Health Organization. Multisystem inflammatory syndrome in children and adolescents with COVID-19. Published May 15,2020

10. Cavalli G, De Luca G, Campochiaro C, Della-Torre E, Ripa M, Canetti D, et al. Interleukin-1 blockade with highdose anakinra in patients with COVID-19, acute respiratory distress syndrome, and hyperinflammation: a retrospective cohort study. Lancet Rheumatol. 2020;2:e325-31. doi: 10.1016/ S2665-9913(20)30127-2

11. Dolinger MT, Person H, Smith R, Jarchin L, Pittman N, Dubinsky MC, et al. Pediatric Crohn's disease and multisystem inflammatory syndrome in children (MIS-C) and COVID-19 treated with infliximab. J Pediatr Gastroenterol Nutr. 2020;2:1535. doi: 10.1097/MPG.0000000000002809

12. Schvartz A, Belot A, Kone-Paut I. Pediatric Inflammatory Multisystem Syndrome and Rheumatic Diseases During SARS-CoV-2 Pandemic. Front. Pediatr. 2020 |https://doi. org/10.3389/fped.2020.605807

13. Belhadjer Z, Méot M, Bajolle F et al. Acute heart failure in multisystem inflammatory syndrome in children (MIS-C) in the context of global SARS-CoV-2 pandemic. Circulation. 2020;33. 10.1161/CIRCULATIONAHA.120.048360 [PubMed]

14. Blondiaux E, Parison P, Redheuil A, et al. Cardiac MRI of children with multisystem inflammatory syndrome (MIS-C) associated with COVID-19: case series Eléonore. Radiology. 2012;78:1-15

15. Grimaud M, Starck J, Levy M et al. Acute myocarditis and multisystem inflammatory emerging disease following SARSCoV-2 infection in critically ill children. Ann Intensive Care. 2020;10: 1-5. https://doi.org/10.1186/s13613-020-00690-8

16. Wolfler A, Mannarino S, Giacomet V et al. Acute myocardial injury: a novel clinical pattern in children with COVID-19. Lancet child Adolesc Heal.2020;4642:1016-1017. https://doi.org/10. 1016/S2352-4642(20)30168-1

17. Rubens JH, Akindele NP, Tschudy MM, Sick-Samuels AC. Acute covid-19 and multisystem inflammatory syndrome in children BMJ 2021;372:n385 doi:10.1136/bmj.n385

18. Pouletty M, Borocco C, Ouldali N, Caseris M, Basmaci $\mathrm{R}$, Lachaume $\mathrm{N}$, et al. Paediatric multisystem inflammatory syndrome temporally associated with SARS-CoV-2 mimicking Kawasaki disease (Kawa-COVID-19): a multicentre cohort. Ann Rheum Dis. 2020;79:999-1006. doi: 10.1136/ annrheumdis-2020-217960

19. Laing AG, Lorenc A, del Molino del Barrio I, Das A,
Fish M, Monin L, et al. A dynamic COVID-19 immune signature includes associations with poor prognosis. Nat Med. 2020; 26:1623-35. doi: 10.1038/s41591-020-1038-6

20. Licciardi F, Pruccoli G, Denina M, Parodi E, Taglietto M, Rosati S, et al. SARS-CoV-2-Induced Kawasaki-like hyperinflammatory syndrome: a novel COVID phenotype in children. Pediatrics. 2020;146:e20201711. doi: 10.1542/peds.2020-1711

21. Pilania RK, Jindal AK, Bhattarai D, Naganur SH, Singh S. Cardiovascular Involvement in Kawasaki Disease Is Much More Than Mere Coronary Arteritis. Front Pediatr. 2020;24;8:526969. doi: 10.3389/fped.2020.526969. PMID: 33072669 ; PMCID: PMC7542237.

22. Cattalini M, Taddio A, Bracaglia C et al. Rheumatology Study Group of the Italian Society of Pediatrics. Childhood multisystem inflammatory syndrome associated with COVID-19 (MIS-C): a diagnostic and treatment guidance from the Rheumatology Study Group of the Italian Society of Pediatrics. Ital J Pediatr. 2021;47(1):24. doi: 10.1186/s13052-021-00980-2. PMID: 33557873; PMCID: PMC7868856.

23. Kochi AN, Tagliari AP, Forleo GB, Fassini GM, Tondo C. Cardiac and arrhythmic complications in patients with COVID-19. J Cardiovasc Electrophysiol. 2020;31(5):1003-1008. doi: 10.1111/jce.14479. Epub 2020 Apr 13. PMID: 32270559; PMCID: PMC7262150.

24. Henderson LA, Canna SW, Friedman KG, Gorelik M, Lapidus SK, Bassiri H, et al. American College of Rheumatology Clinical Guidance for Pediatric Patients with Multisystem Inflammatory Syndrome in Children (MIS-C) Associated with SARSCoV-2 and Hyperinflammation in COVID-19. Version 1. Arthritis Rheumatol 2020;72:1791-1805. doi: https://onlinelibrary. wiley.com/doi/10.1002/art.41454.

25. Feldstein LR, Rose EB, Horwitz SM, Collins JP, Newhams MM, Son MBF, et al. Multisystem inflammatory syndrome in U.S. children and adolescents. N Engl J Med. 2020;383:33446. doi: 10.1056/NEJMoa2021680

26. Wright FL, Vogler TO, Moore EE et al. Fibrinolysis shutdown correlates to thromboembolic events in severe COVID-19 infection. J Am Coll Surg. 2020:1-11. 10.1016/j.jamcollsurg.2020.05.007 [PMC free article] [PubMed]

27. Esposito, S., Principi, N. Multisystem Inflammatory Syndrome in Children Related to SARS-CoV-2. Pediatr Drugs. 2021; 23:119-129. https://doi.org/10.1007/s40272-020-00435-x 